\title{
DNAJB1/PRKACA Fusion Gene
}

National Cancer Institute

\section{Source}

National Cancer Institute. DNAIB1/PRKACA Fusion Gene. NCI Thesaurus. Code C122558.

A fusion gene that results from a heterozyg ous deletion in chromosome 19 that fuses the first 1 or 2 exons of the DNAJB1 gene in frame with exon 2 of the PRKACA gene. This fusion is associated with fibrolamellar carcinoma. 